\title{
Design and Performance of the Douglas Mawson Telescope
}

\author{
Jon S. Lawrence, Michael C. B. Ashley, Michael G. Burton and John W.V. Storey \\ School of Physics, University of New South Wales, Sydney, NSW 2152 Australia
}

\begin{abstract}
The Douglas Mawson Telescope (DMT) is a proposed $2 \mathrm{~m}$ telescope to be situated on the Antarctic plateau. The proposal comes from Australia, and invites participation by other nations, especially those already active in Antarctic astronomy; such as Italy, France and the United States. The DMT will be equipped with instrumentation to perform wide-field imaging from the near to far infrared. Results from an extensive site testing campaign over the last decade indicates that an Antarctic infrared telescope can be one to two orders of magnitude more sensitive than any other ground based telescope of the same size. The DMT will be an important tool for astrophysical research. It will also be beneficial as a technological test bed for future large (8-10 m class) Antarctic telescopes and interferometers, and for space-based telescopes. This paper analyses the performance of the DMT in terms of the achievable resolution, field-ofview, sensitivity and survey depth and compares it to a similar sized telescope located with the characteristic midlatitude atmosphere of Mauna Kea.
\end{abstract}

Keywords: astronomical optics, atmospheric turbulence, site testing, Antarctic astronomy, adaptive optics

\section{INTRODUCTION}

The first phase of the development of the Antarctic plateau as an astrophysical observatory commenced and is continuing with an extensive range of site testing experiments characterising the atmosphere. These site testing experiments have indicated that Antarctica is arguable the best location on Earth to place an infrared or submillimetre telescope. The superiority of the site arises from several factors: the very low temperatures, which result in low thermal background radiation and telescope emission; the low water vapour content in the atmosphere, which decreases atmospheric absorption; the seeing caused by atmospheric turbulence, which although moderate compared to midlatitude temperate sites, is mostly confined to the inversion layer close to the ground; and the long Antarctic night and low cloud cover, which indicate that long observation periods should be possible.

The second phase in the development of an Antarctic observatory consisted of the design and construction of the prototype near infrared $600 \mathrm{~mm}$ SPIREX/ Abu telescope, and is currently continuing with the development of the mid infrared $800 \mathrm{~mm}$ IRAIT telescope. The SPIREX telescope successfully demonstrated the feasibility of Antarctic winter operation at the South Pole and drew attention to relevant design issues. Additionally, although SPIREX was a moderate sized telescope, due to the superior site conditions observations have led to a number of publications in the field of star formation. The IRAIT telescope will demonstrate operation at Dome C, a more remote and potentially superior site to the South Pole.

This paper analyses performance goals for the next generation of Antarctic telescopes, which are expected to have $\mathrm{a} \sim 2 \mathrm{~m}$ main mirror diameter. Currently, there are two proposals for such an instrument: the DMT (an Australian, Italian, French, and US collaboration) and the AIRO (a US proposal). The results of the site testing program to-date have been used to estimate the performance capabilities of such a telescope. Firstly, the achievable resolution of the DMT is modeled as a function of wavelength based on Antarctic turbulence data. This analysis considers that the telescope will be equipped with a tip-tilt secondary mirror. Secondly, these results are used to determine appropriate field-of-views with existing CCD technology. Thirdly, atmospheric transmission and emission data is used to determine the limiting magnitude as a function of wavelength. This is then used to determine the survey depth capabilities over time. In each case the average and $25 \%$ best South Pole atmospheres are considered and compared to the average Mauna Kea atmosphere. Survey depth comparisons are also made with the Next Generation Space Telescope.

*j1@phys.unsw.edu.au; phone 6129385 5003; fax 6129385 6060; http://www.phys.unsw.edu.au/astro; University of New South Wales, Sydney NSW 2052, Australia 


\section{SITE TESTING RESULTS}

A number of groups have been actively involved in site testing the applicability of the Antarctic atmosphere for astronomical observations. These include the Joint Australian Centre for Astrophysical Research in Antarctica (JACARA), the French Istitut polaire francais Paul-Emile Victor (IPEV), the Italian Programma Nazionale di Ricerche in Antartide (PNRA), and the US Center for Astrophysical Research in Antarctica (CARA). Data has been collected on various atmospheric characteristics: the turbulence structure, the infrared sky background, the cloud cover statistics, and the sub-millimetre opacity and sky temperature.

Atmospheric turbulent studies have been conducted primarily at the US Amudsen-Scott South Pole station. They include an extensive series of meteorological and microthermal balloon launches ${ }^{1}$. Additionally, as part of the Automated Astrophysical Site Testing Observatory ${ }^{2}$ (AASTO) two instruments have been operated: the ADIMM $^{3}$ (a differential image motion monitor) and the $\operatorname{SODAR}^{4}$ (an acoustic sound and ranging antenna). These have determined the average South Pole ground level seeing to be $\sim 1.6 \operatorname{arcsec}$ at $0.5 \mu \mathrm{m}\left(\mathrm{r}_{0}=0.064 \mathrm{~m}\right)$. They have also discovered the unique nature of atmospheric turbulence above the South Pole site, ie it consists of a strong turbulent layer close to the ground (typically within $300 \mathrm{~m}$ ) but with very little high altitude turbulence. This results in an isoplanatic angle as large as 1 arcmin, which is significantly larger than typical of mid-latitude sites. Turbulent profiles determined from balloon launch data are currently being collected and analysed from the Italian/French Dome Concordia station. The SODAR is planned for deployment to Dome C in 2003, as part of the Automated Astrophysical Site Testing INfant Observatory (AASTINO), and several differential image motion monitor experiments are planned for the future.

Observations of the infrared sky spectral brightness have been made from the South Pole station with several instruments: the GRISM (a near infrared grism spectrometer) on the SPIREX telescope ${ }^{5}$, the Infrared Photometer Spectrometer $^{6}$ (IRPS), the Mid-infrared Sky Monitor ${ }^{7}$ (MISM), and the Near Infrared Sky Monitor ${ }^{8}$ (NISM). These instruments cover the 1.5 to $15 \mu \mathrm{m}$ waveband and have shown that the South Pole sky background is up to two orders of magnitude lower than observed at typical mid-latitude sites. The NISM and the MISM are planned for deployment to Dome $\mathrm{C}$ in the near future as part of the AASTINO.

Antarctic site testing at sub-millimetre wavelengths has consisted of the operation of two instruments at the South Pole: the UNSW SUMMIT (a tipping radiometer) and the NRAO/CMU sub-millimetre tipper (both are of similar construction). These instruments have shown that the average South Pole opacity at $350 \mu \mathrm{m}$ is significantly less than that observed at Mauna Kea and Chajnantor and has a much lower variance. This has motivated the development of the AST/RO sub-millimetre telescope at the South Pole station ${ }^{9}$. Additionally the SUMMIT was in operation at Dome C over the 2000-2001 summer, and demonstrated an average opacity significantly lower than that at South Pole over the same period ${ }^{10}$.

Balloon borne radiosonde measurements have determined that the water vapour content of the Antarctic atmosphere is lower than observed at any other location ${ }^{11}$. This implies that the atmospheric transmission will be higher for sites on the Antarctic plateau than any other ground-based site for wavelengths where water vapour dominates the atmospheric absorption. Atmospheric transmission measurements, and models based on the determined water vapour content indicate that while there is a noticeable improvement at near to mid infrared wavebands the most significant effect occurs at longer wavelengths including the sub-millimetre.

Cloud cover statistics have been taken over the last decade from the South Pole station. These have shown that on average the South Pole sky is completely cloud free for $\sim 30 \%$ of the time, although these are daily approximations from subjective observations only. Two cloud monitoring instruments (ICECAM and COBBER) are currently in operation at Dome $\mathrm{C}$ and indicate that the sky is cloud free for as much as $80 \%$ of the time, although more data need to be collected for a more accurate statistical average. 


\section{RESOLUTION}

The average seeing through the South Pole atmosphere $(1.6 \operatorname{arcsec}$ at $0.5 \mu \mathrm{m})$ determined from the value of the atmospheric coherence length (from site testing studies), is significantly inferior to the average seeing of very good quality mid-latitude site such as Mauna Kea $(0.5 \mathrm{arsec}$ at $0.5 \mu \mathrm{m})$. This value is often used as a representation of the quality of a particular site for astronomical observations. However, for a telescope equipped with any sort of adaptive optics correction (including low-order tip-tilt correction for which the DMT will be equipped) this value should not be considered in isolation, as many other factors determine the achievable resolution with an adaptive optics system. An analysis of the errors associated with adaptive optics correction on Antarctic telescopes is given in reference (12), both for a $2 \mathrm{~m}$ tip-tilt telescope and for an $8 \mathrm{~m}$ telescope equipped with high-order adaptive optics. The $2 \mathrm{~m}$ analysis and results are outlined here. They are based on models of the refractive index structure constant profile obtained from the SODAR experiment and wind velocity profiles obtained from meteorological balloon borne experiments.

A tip-tilt system corrects the low order atmospheric turbulence induced phase variance of an image of an astronomical object by measuring the tip and tilt fluctuations of the light from a guide star (of a particular magnitude and angular separation from the object) and correcting this phase variance with a tiltable mirror (usually the secondary). The resolution achievable with a tip-tilt corrected telescope depends on a number of errors which are introduced in the wavefront correction process. These errors are: residual high order error, tilt anisoplanatic error, signal to noise ratio error, bandwidth error, and centroid anisoplanatic error; see ref (13) for an analysis of these errors. The residual error arises from higher-order modes which are not corrected by the tip-tilt mirror; it represents $\sim 30 \%$ of the uncompensated atmospheric phase variance. The tilt anisoplanatic error arises because of the finite angular distance between the object to be imaged and the guide star. Light from both traverses different paths through the atmosphere and thus experiences a different total phase variance. The error depends on the atmospheric turbulence profile above the particular site, and scales with the separation of the object star to the guide star. The signal to noise ratio error arises due to the finite signal to noise ratio in the tilt detection system. It depends on the magnitude of the guide star and various properties of the wavefront sensor. The fourth error is due to the finite bandwidth of the detection system. This arises because there is a temporal delay between the detection of the guide star wavefront and the correction of the object wavefront. The centroid anisplanatic error arises because the wavefront sensor measures a variance with contributions from high-order modes.

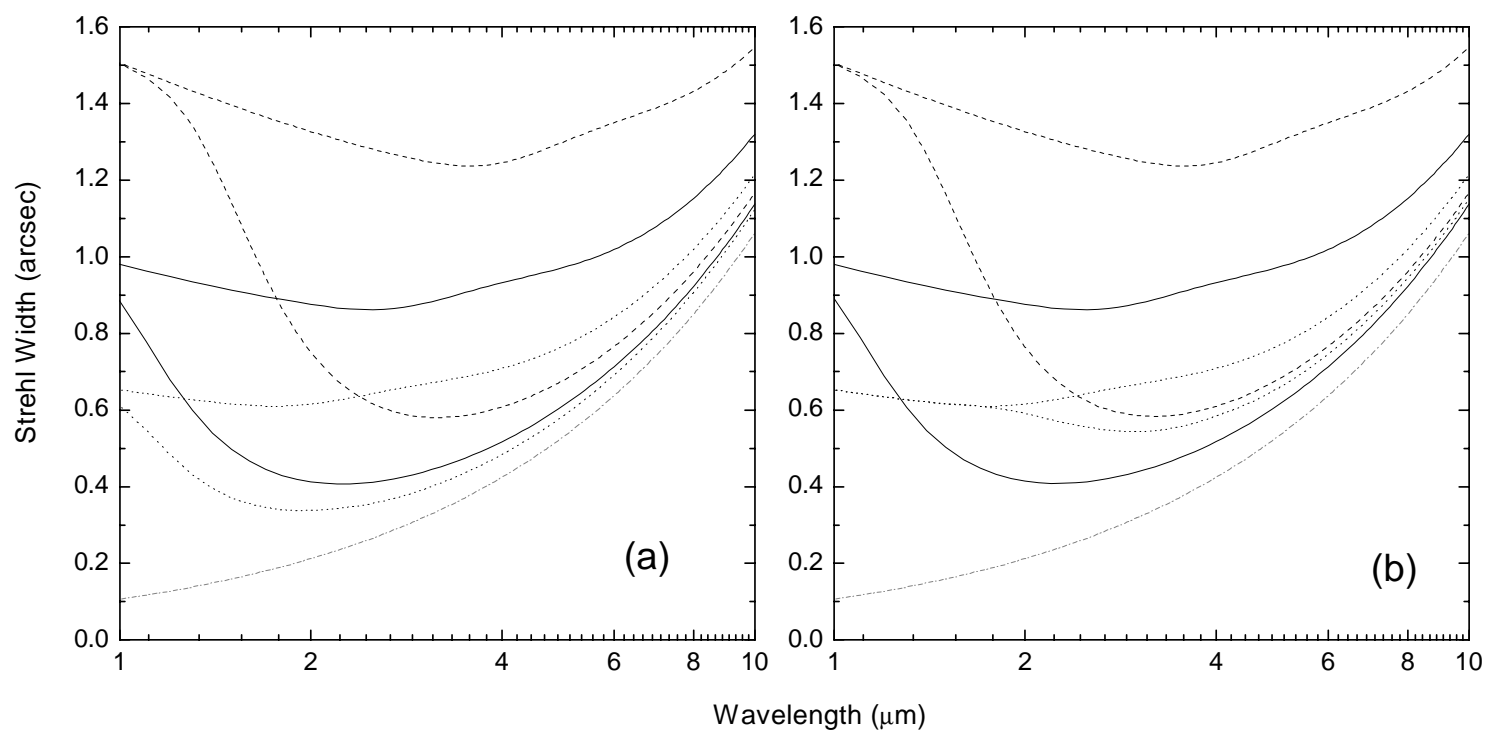

Figure 1. Strehl width achievable with a $2 \mathrm{~m}$ telescope versus wavelength for average South Pole (dashed line), 25\% best South Pole (solid line), and average Mauna Kea (dotted line) atmospheric conditions. Graph (a) is for observations of the galactic plane, and graph (b) is for observations of the galactic pole. In each case the uncompensated atmosphere resolution (upper curve) and tip-tilt corrected resolution (lower curve) is shown. The diffraction limited Strehl width is also shown (dash-dot line). 
In order to determine the efficiency of a tip-tilt correction the total phase variance due to the total wavefront error must be calculated. Generally, there is a combination of guide star magnitude and guide star separation that can be found which minimises the total error. This occurs because the combined signal-to-noise ratio and bandwidth errors increase with the magnitude of the tip-tilt guide star (due to the decreased SNR), whereas the tilt anisoplanatic error increases with the angular separation of the object and guide stars. As the angular separation increases, the probability of finding a star of brighter magnitude also increases. This probability, which represents the fraction of the sky that has coverage for a given stellar magnitude, is termed the sky coverage factor. It has a different functional form for observations of the galactic plane and the galactic pole.

Figure 1 shows the variation of the Strehl width for a $2 \mathrm{~m}$ telescope as a function of wavelength for average and $25 \%$ best South Pole atmospheric conditions and for average Mauna Kea atmospheric conditions, for a sky coverage factor of $90 \%$. In either the galactic plane or the galactic pole the resolution achievable for tip-tilt correction for the South Pole telescope is determined almost entirely by the higher-order residual error. Due to the large isoplanatic angle, the anisoplanatic errors increase with angular separation at a rate slow enough that very bright stars can always be used, such that the anisoplanatic and signal-to-noise ratio errors are negligible compared with the residual error. This is also the case for the Mauna Kea telescope observing in the galactic plane. For Mauna Kea observations of the galactic pole, however, the sparse stellar distribution combined with the increased anisoplanatic error gradient results in a minimum error that degrades the resolution because much fainter stars at smaller angular separations have to be used as guide stars. This degradation is such that for observation above $1.2 \mu \mathrm{m}$ a superior resolution is attainable by the South Pole telescope for the best $25 \%$ of observing conditions.

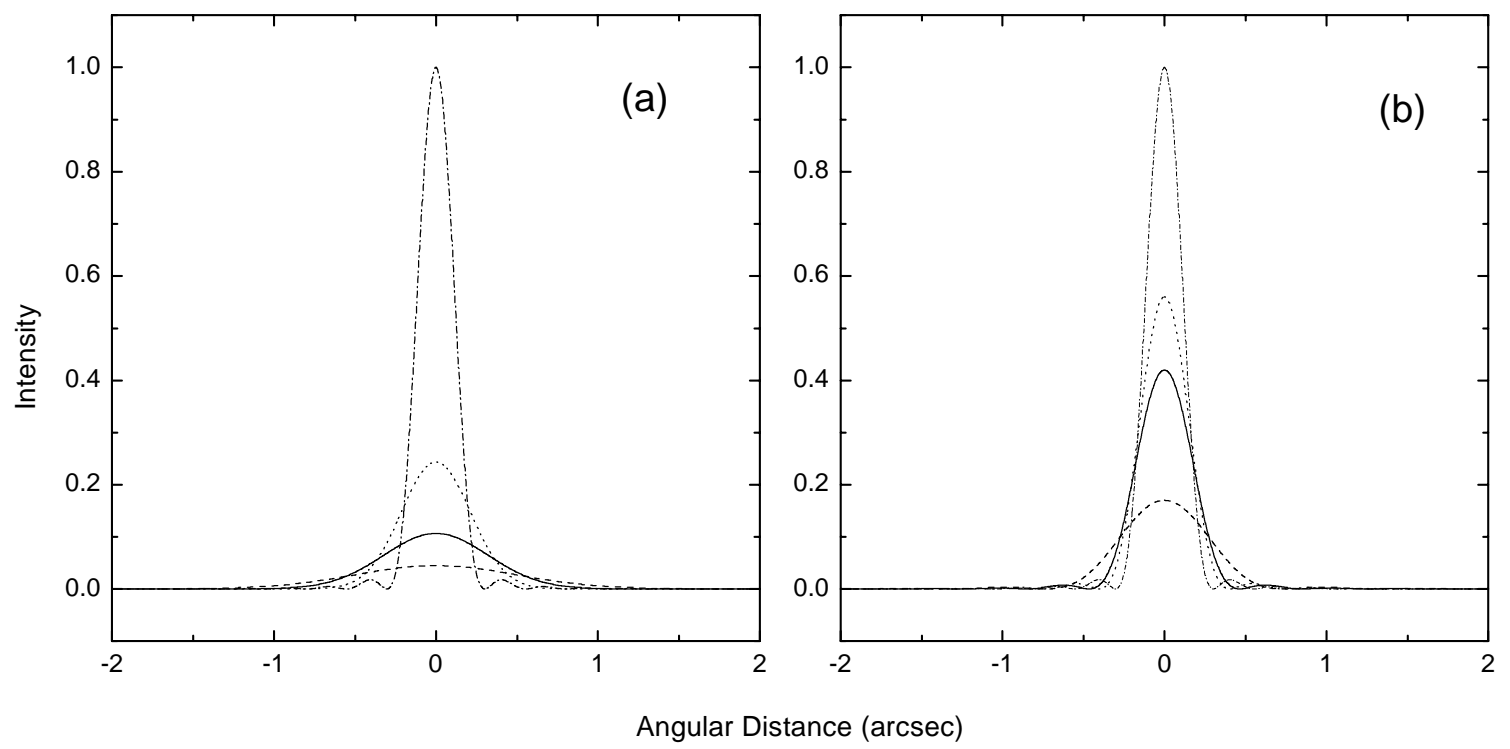

Figure 2. $2.4 \mu \mathrm{m}$ diffraction limited point spread function for a $2 \mathrm{~m}$ telescope (dash-dot line) compared to the uncompensated atmospheric point spread function (in (a)) and the tip-tilt removed point spread function (in(b)) for average South Pole conditions (dashed line), 25\% best South Pole conditions (solid line) and average Mauna Kea conditions (dotted line).

The simulated atmospheric point spread function is shown compared to the diffraction limit in figure 2 (a) for the average South Pole, $25 \%$ best South Pole, and average Mauna Kea atmospheres for a wavelength of $2.4 \mu \mathrm{m}$. These figures represent Strehl ratios of 0.04, 0.11, and 0.24 for each atmosphere respectively. The effect of tip-tilt correction for each atmosphere is shown in figure 2 (b). The Strehl ratios are improved to $0.17,0.42$, and 0.56 respectively. The figure demonstrates the significant increase in the resolution that can be obtained with tip-tilt correction alone. For wavelengths longer than $5 \mu \mathrm{m}$ near diffraction limited resolution (Strehl ratio > 0.5) is achievable for the average South Pole atmosphere, and near diffraction limited observation is possible longward of L band for the best $25 \%$ of observing conditions. 


\section{FIELD OF VIEW}

Based on the analysis of the DMT tip-tilt corrected resolution, the achievable field of view can be determined appropriate to current CCD technology. The pixel scale chosen depends on a number of trade-offs. Firstly, the resolution is inversely proportional to the field of view at any particular wavelength for a given detector array. Secondly, increasing the field of view decreases the photometric accuracy if the focal plane point spread function is not correctly sampled. Finally, there is a trade-off between the average resolution achievable due to variability in atmospheric conditions.

The design goal for the DMT imaging camera must be primarily motivated towards a wide field of view, consistent with science goals of performing wide field sky surveys ${ }^{14}$. However, the highest possible resolution is also required in order that multi-waveband comparisons with data collected from other large telescopes is meaningful. This resolution will be far below that which can be achieved with a space-based telescope, but will be similar to that achievable with near and mid-infrared cameras operated on large 8-10 m mid-latitude telescopes (without adaptive optics). For imaging at the Nyquist sampling rate required to give a photometric accuracy of $\sim 1 \%$, ie 2.5 pixels per FWHM of the atmospheric point spread function, the field-of-view for a $2 \mathrm{~m}$ telescope is seriously degraded. Recent image restoration and reconstruction techniques, such as Dithering and drizzling, allow the resolution to be improved for an undersampled image. These techniques, however, rely on a point spread function that does not vary in the telescope focal plane, ie it has so far only been proved efficient for space based telescopes imaging at the diffraction limit ${ }^{15}$. The effectiveness of such techniques should improve for adaptive optics corrected imaging. However, for partial correction, such as is proposed for the tip-tilt secondary DMT, the efficiency of such techniques is not yet determined.

Table1 shows the field of view achievable for the DMT with two different pixel scales in three different wavebands. The first pixel scale for each waveband is for an undersampled image at $\sim 1$ FWHM of the corrected point spread function per pixel, and the second pixel scale is for a Nyquist sampled image at $\sim 2.5$ FWHM of the corrected point spread function. The FWHM of the corrected point spread function is weighted towards the $25 \%$ best South Pole atmospheric observing conditions because such conditions are considered to comprise the majority of observing time, ie neglecting very high turbulence conditions from the total average. The table shows that significant field of views can be obtained (20 armin at $2.4 \mu \mathrm{m})$ but only for undersampled images.

Two possible types of imaging camera are considered appropriate for the DMT. The first uses a single array detector in the telescope focal plane. This camera is envisaged for its simplicity- ie, the only optics it would require is a single field flattener in front of the CCD dewar. The focal plane scale for a $2 \mathrm{~m}$ telescope can be appropriately designed for any of the pixel scales shown in table $1^{16}$. The second camera is a multi-colour imaging camera with three (or four) detectors, probably imaging in each of the wavebands shown in Table 1. For each case only a single array detector is considered although mosaicing several detectors could significantly increase the field of view. For each detector array, the largest currently available size is considered.

Table 1. Field of view (FOV) achievable with the DMT in three different wavebands for different pixel scales.

\begin{tabular}{ccccc}
\hline Waveband $(\mu \mathrm{m})$ & Array size & Detector & $\begin{array}{c}\text { Pixel scale } \\
\left(\operatorname{arcsec}_{\text {pixel }}{ }^{-1}\right)\end{array}$ & FOV (arcmin) \\
\hline $1-2.5$ & $2056 \times 2056$ & HgCdTe & 0.6 & 20.5 \\
& & & 0.24 & 8.22 \\
$2.5-5$ & $1024 \times 1024$ & HgCdTe/ InSb & 0.7 & 12.0 \\
& & & 0.28 & 4.7 \\
$5-15$ & $512 \times 512$ & $\mathrm{Si}:$ As & 1.2 & 10.2 \\
& & & 0.48 & 4.0 \\
\hline
\end{tabular}




\section{SENSITIVITY}

Of primary importance to characterise the performance of any infrared site is the sensitivity achievable for any astronomical observation. The sensitivity is determined by the signal to noise ratio obtained for observations of a particular stellar magnitude over a given integration time. The signal to noise ratio depends on a number of factors. Firstly, and most significant for background limited observations in the infrared, are the atmospheric transmission and emission at the particular site. Secondly, the pixel scale is important because it determines the signal strength per pixel and the total background flux. Finally, parameters of the detector array must be considered.

The limiting magnitude for a signal to noise ratio of 5 with an hour of integration is shown in figure 3 as a function of wavelength. A $2 \mathrm{~m}$ telescope is considered operating under three different atmospheric conditions: the average South Pole atmospheric transmission and emission, the South Pole atmospheric transmission and emission for very clear conditions, and the average Mauna Kea atmospheric transmission and emission. South Pole and Mauna Kea transmission are taken from an ATRAN model based on water vapour column densities of $500 \mu \mathrm{m}$ and $1500 \mu \mathrm{m}$ respectively. South Pole sky background is from a combination of IRPS ${ }^{6}$ and MISM $^{7}$ data and a MODTRAN atmospheric model. Mauna Kea sky background is taken from an ATRAN atmospheric model with standard conditions ${ }^{17}$. In each case the pixel scale is 0.6 arcsec per pixel, the CCD read noise is 5 electrons and the CCD dark current is 1 electron. $\mathrm{s}^{-1}$.

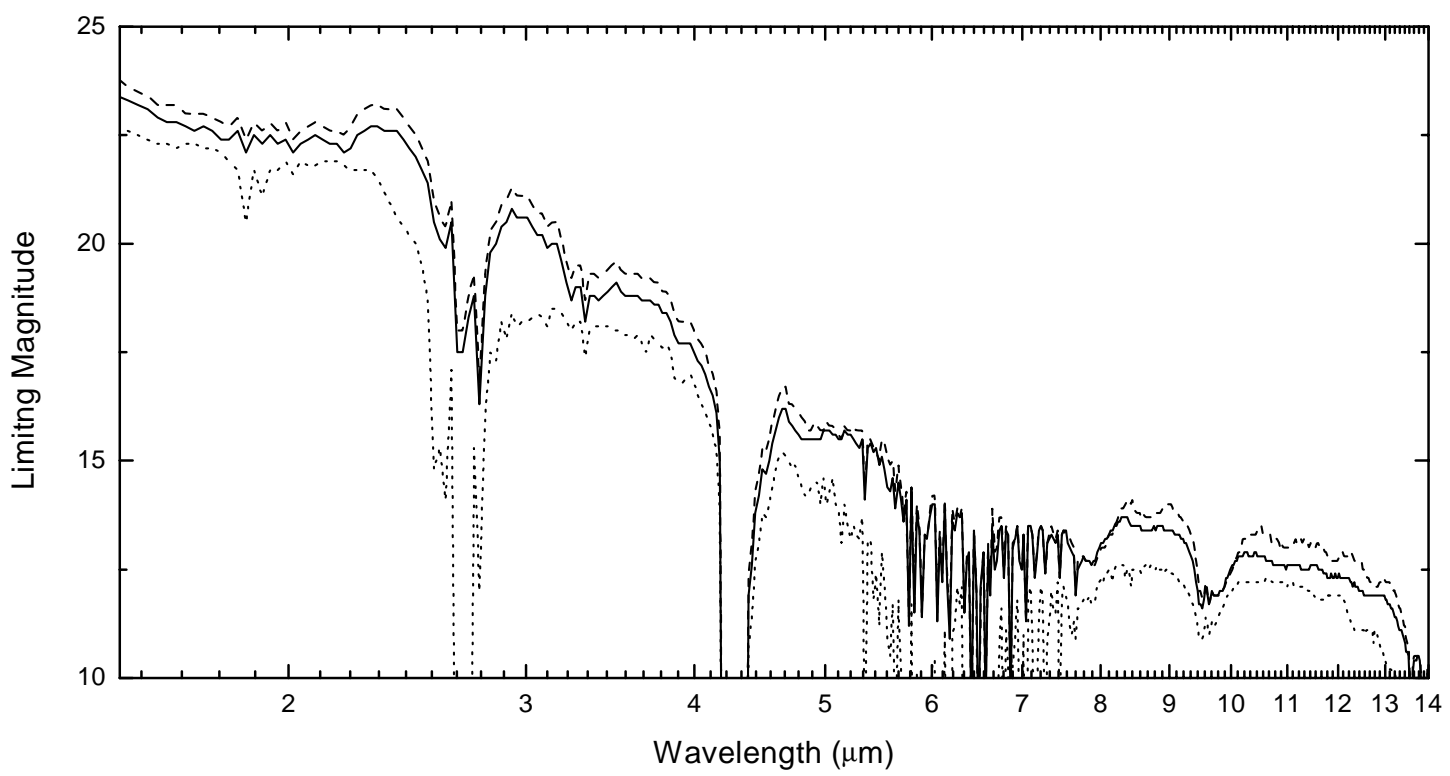

Figure 3. Limiting magnitude as a function of wavelength for the DMT under clear South Pole conditions (solid line), and for very clear South Pole conditions (dashed line) compared to a $2 \mathrm{~m}$ telescope operated at Mauna Kea (dotted line). Data includes the atmospheric transmission and emission.

The DMT should be capable of reaching 23 magnitude in $\mathrm{K}$ band and $\sim 13$ magnitude in $\mathrm{N}$ band for an hour of integration during the best observing conditions. Due to the significantly lower sky emission, the limiting magnitude for a $2 \mathrm{~m}$ South Pole telescope throughout the near and mid infrared is between one and two magnitudes fainter than for a similar telescope located at Mauna Kea. This represents a significant increase; one magnitude represents a factor of 10 increase in collecting area, and thus the DMT is equivalent in sensitivity to an $8 \mathrm{~m}$ mid-latitude telescope. Additionally, there are a number of regions, such as between $\mathrm{K}$ and $\mathrm{L}$ bands at $\sim 2.8 \mu \mathrm{m}$, and between $\mathrm{M}$ and $\mathrm{N}$ bands at 5.5-7.5 $\mu \mathrm{m}$, where observation from most ground-based sites is not possible due to strong atmospheric water vapour absorption. At the South Pole bright stars can readily be observed at these wavelengths. This model considers point source observations. For observations of extended objects the relative increase in South Pole sensitivities compared with Mauna Kea sensitivities is similar. 


\section{SURVEY DEPTH}

One of the primary science goals for the DMT is to perform sky surveys in the near and mid infrared. For this reason the survey depth achievable should be characterised. This represents the area of sky that can be observed in a given survey time at a particular stellar magnitude. Figure 4 shows the stellar magnitude versus sky coverage factor achievable for a signal to noise ratio of 5 with the DMT in a year of total observation time with clear and very clear conditions at the South Pole, compared to a similar $2 \mathrm{~m}$ survey telescope operated at Mauna Kea. Comparison is also made with the survey depth achievable with the Next Generation Space Telescope. Both $2.4 \mu \mathrm{m}$ and $11 \mu \mathrm{m}$ operation is shown. For the $2 \mathrm{~m}$ telescope the field of view is as defined in table 1 (ie $24 \operatorname{arcmin}$ at $2.4 \mu \mathrm{m}$ and $8 \operatorname{arcmin}$ at $11 \mu \mathrm{m}$ ). The NGST, with a $6 \mathrm{~m}$ main mirror diameter, is considered to have a $2.3 \operatorname{arcmin}$ field of view at $2.4 \mu \mathrm{m}$ and a 3 arcmin field of view at $11 \mu \mathrm{m}^{18}$. This represents a mosaic of $2048 \times 2048$ arrays at the low wavelength end. For each curve the lower sky coverage limit represents the detector field of view. The upper sky coverage limit represents a single field of view integration time of 10 seconds, ie allowing for re-pointing and calibration of the telescope.
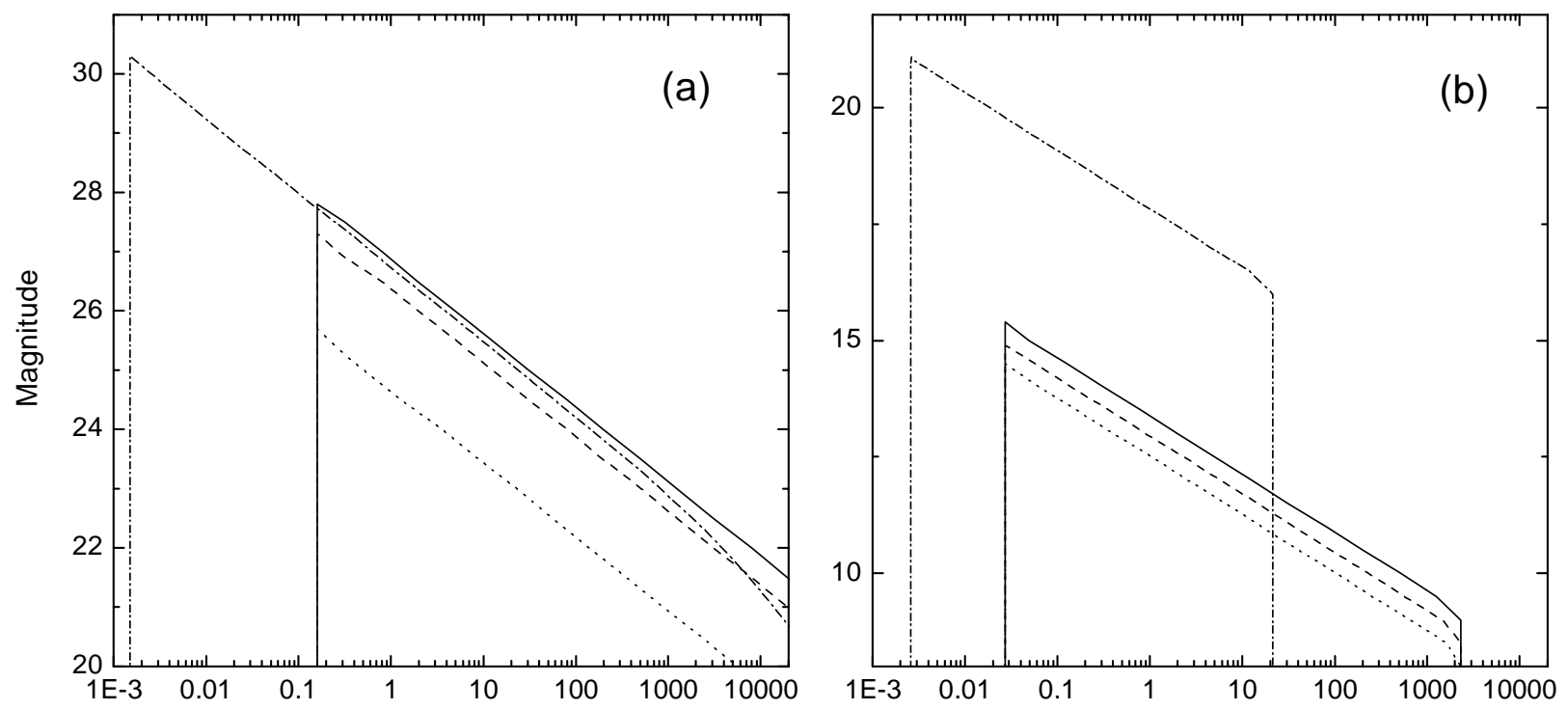

Sky Coverage $\left(\right.$ degree $\left.^{2}\right)$

Figure 4. Survey depth (stellar magnitude versus sky coverage) achievable in a year of observation for: the DMT under average South pole conditions (dashed line) and very clear South Pole conditions (solid line), a $2 \mathrm{~m}$ telescope located at Mauna Kea (dotted line), and the NGST (dash-dot line). (a) shows operation at $2.4 \mu \mathrm{m}$ and (b) shows operation at $11 \mu \mathrm{m}$.

Figure 4 shows that the DMT should be able to cover the observable sky in the $\mathrm{K}_{\mathrm{dark}}$ band $(2.4 \mu \mathrm{m})$ out to a magnitude of $\sim 21$ in a year of integration time. In $\mathrm{N}$ band a significant portion of the sky can be covered out to $\sim 10$ magnitude in a year. As expected for any other ground-based site the time necessary to complete a given survey is severely increased. A Mauna Kea $2 \mathrm{~m}$ telescope requires $\sim 10$ years to complete such a survey. Comparisons with the NGST survey depth indicate the complementary nature of the DMT science goals. Given the far superior resolution and sensitivity of the NGST its primary role is for very deep imaging of narrow regions. The DMT can complement such studies with wide field of view, less deep observations for which it can dedicate significant amounts of time.

A factor not included in this analysis is cloud cover statistics. This awaits more detailed site-testing data. Although the South Pole cloud cover is quite high compared to such sites as Mauna Kea, due to the low sky background there is still a benefit in terms of total observing time required for a particular survey. A complete comparison of the achievable survey depth at Mauna Kea with South Pole (and also Dome C) requires the total average amount of useful observation time at each location. This must also include an analysis of the effects of twilight sky emission and sky emission in the presence of moonlight for each location. 


\section{CONCLUSION}

In summary, an analysis has been presented of the achievable design goals for the Douglas Mawson Telescope, a $2 \mathrm{~m}$ infrared telescope to be located on the Antarctic plateau. This has shown that tip-tilt corrected resolution of this telescope should be close to that achievable from the best mid-latitude astronomical observatory sites for the best atmospheric conditions. The sensitivity of the DMT should be significantly better than a similar telescope located at any other ground-based site, and thus the DMT should be capable of performing sky surveys over large areas of the sky an order of magnitude quicker than any other infrared ground-based survey telescope.

The data here represent models of performance based on the atmospheric conditions encountered at the South Pole. There is reason to believe that the atmospheric conditions at Dome $\mathrm{C}$ are even more appropriate for infrared astronomical observations. This benefit will be quantified in the near future by the extension of a series of site testing experiments from South Pole to Dome C station.

\section{ACKNOWLEDGEMENTS}

The authors wish to thank the University of New South Wales (UNSW) Antarctic astronomy group, the French Istitut polaire francais Paul-Emile Victor (IPEV), the Italian Programma Nazionale di Ricerche in Antartide (PNRA), and the US Center for Astrophysical Research in Antarctica (CARA) for collaboration in many areas of site testing. Additionally, we thank the Australian Research Council and the Australian Antarctic Division for funding, and the US National Science Foundation for logistic support of our site testing campaigns.

\section{REFERENCES}

1. R. D. Marks, J. Vernin, M. Azouit, J. W. Briggs, M. G. Burton, M. C. B. Ashley, and J. F. Manigault, "Antarctic site testing - microthermal measurements of surface-layer seeing at the South Pole," Astron. Astrophys. Suppl. Ser. 118, pp. 385-390, 1996.

2. J. W. V. Storey, "The AASTO program," in Astrophysics from Antarctica, ed. R. Landsberg and G. Novak ASP Conf. Ser. 141, pp. 313-218, ASP, San Fransisco, 1998.

3. M. A. Dopita, P. R. Wood, and G. R. Hovey, "An automated DIMM telescope for Antarctica," Publ. Astron. Soc. Aust. 13, pp. 13-43, 1996.

4. T. Travouillon, M. C. B. Ashey, M. G. Burton, J. W. V. Storey, and R. F. Lowenstein, "Atmospheric turbulence at the South Pole and its implications for astronomy," submitted to Aston. Astrophys. 2002.

5. M. Herald, "SPIREX - near infrared astronomy from the South Pole," Exp. Astron. 3, pp. 87-91, 1994.

6. A. Phillips, M. G. Burton, M. C. B. Ashley, J. W. V. Storey, J. P. Lloyd, D. A. Harper, and J. Bally, "The near infrared sky emission at the South Pole in winter," Astrophys. J. 527, pp. 1009-1022, 1999.

7. M. A. Chamberlain, M. C. B. Ashley, M. G. Burton, A. Phillips, J. W. V. Storey, and D. A. Harper, "Mid-infrared observing conditions at the South Pole," Astrophys. J. 535, pp. 501-511, 2000.

8. J. S. Lawrence, M. C. B. Ashley, M. G. Burton, P. G. Calisse, J. R. Everett, R. J. Pernic, A. Phillips, and J. W. V. Storey, "Operation of the Near Infrared Sky Monitor at the South Pole," submitted to Publ. Astron. Soc. Aust., 2001.

9. A. A. Stark, R. A. Chamberlain, G. Ingalis, J. Cheng, and G. Wright, "Optical and mechanical design of the Antarctic Submillimeter Telescope and Remote Observatory," Rev. Sci. Instr. 68, pp. 2200-2213, 1997.

10. P. G. Calisse, J. W. V. Storey, M. C. B. Ashley, M. G. Burton, S. Radford, and J. Peterson, "Sub-millimetre site testing at Dome C," in preparation, 2002.

11. A. P. Lane, "Submillimeter transmission at the South Pole," in Astrophysics from Antarctica, ed. R. Landsberg and G. Novak ASP Conf. Ser. 141, pp. 289-295, ASP, San Fransisco, 1998.

12. J. S. Lawrence, "Adaptive optics performance for Antarctic telescopes," submitted to J.Opt. Soc. Am. A, 2002.

13. S. S. Olivier and D. T. Gavel, "Tip-tilt compensation for astronomical imaging," J. Opt. Soc. Am. A, 11, pp. 368378, 1994.

14. M. G. Burton, J. W. V. Storey, and M. C. B. Ashley, "Science goals for an Antarctic infrared telescope," Publ. Astron. Soc. Aust. 18, pp.1-8, 2000. 
15. G. Bernstein, "Advanced exposure-time calculations: undersampling, dithering, cosmic rays, astrometry, and ellipticities," Publ. Astron. Soc. Pac. 114, pp. 98-111, 2002.

16. P. R. Gillingham, "An optical design for an Antarctic $2 \mathrm{~m}$ telescope with a near IR imager," Publ. Astron. Soc. Aust. 19, pp. 301-305, 2002.

17. P. Puxley, T. V. Hippel, and T. Geballe, “Observing constraints - sky background”, Gemini Home Page, http://www.gemini.edu/sciops/ObsProcess/ObsProcIndex.html, 2001.

18. G. S. F. Center, "Technology development requirements and goals for the NGST detectors," Next Generation Space Telescope integrated science instrument module, http://www.stsci.edu/ngst/instruments/isim, 2001. 